CERN-PH-TH/2005-190

IFT-UAM/CSIC-05-41

hep-th/0510073

\title{
From F/M-theory to K-theory and back
}

\author{
Iñaki García-Etxebarria ${ }^{\dagger}$, Angel M. Uranga ${ }^{\ddagger}$ \\ † Instituto de Física Teórica, C-XVI \\ Universidad Autónoma de Madrid \\ Cantoblanco, 28049 Madrid, Spain \\ $\ddagger$ TH Unit, CERN, \\ CH-1211 Geneve 23, Switzerland \\ innaki.garcia@uam.es, angel.uranga@cern, ch
}

\begin{abstract}
We consider discrete K-theory tadpole cancellation conditions in type IIB orientifolds with magnetised 7-branes. Cancellation of K-theory charge constrains the choices of world-volume magnetic fluxes on the latter. We describe the F-/M-theory lift of these configurations, where 7-branes are encoded in the geometry of an elliptic fibration, and their magnetic quanta correspond to supergravity 4 -form field strength fluxes. In a K3 compactification example, we show that standard quantization of 4-form fluxes as integer cohomology classes in K3 automatically implies the K-theory charge cancellation constraints on the 7-brane worldvolume magnetic fluxes in string theory (as well as new previously unnoticed discrete constraints, which we also interpret). Finally, we show that flux quantization in F-/M-theory implies that 7-brane worldvolume flux quantization conditions are modified in the presence of 3-form fluxes.
\end{abstract}




\section{Introduction}

One of the important insights into the properties of D-branes is that their charges are classified by K-theory [1, 2]. K-theory charges differ from cohomology charges in discrete pieces, which typically correspond to charges of stable non-BPS D-branes [3].

An interesting consequence of the K-theory classification of D-brane charge for string compactification, is that the consistency conditions should involve cancellation of RR charge in K-theory, rather than in cohomology [4]. This leads to additional discrete constraints, which are related to cancellation of global anomalies in the effective lower-dimensional theory, or on suitable D-brane probes [4]. These discrete constraints lead to non-trivial conditions in diverse compactifications with D-branes (see e.g. $[5,6]$ ) and even play an interesting role in building phenomenologically realistic models in string theory $[7,8]$.

It is convenient to understand K-theory charge cancellation conditions from different viewpoints. This is important, since they are not detectable by standard techniques, e.g. the computation of RR tadpoles via factorization of amplitudes reproduces only $\mathbf{Z}$-valued charges. Moreover, their proper geometric interpretation seems to require the interpretation of RR fields in K-theory [9]. As mentioned above, a possibility is to relate them to cancellation of global anomalies on the world-volume of suitable probes [4].

In this paper we address these consistency conditions from a different viewpoint. We consider a type IIB orientifold compactification with O7-planes and magnetised D7-branes, namely D7-branes carrying non-trivial abelian world-volume gauge bundles. The model has non-trivial K-theory $\mathbf{Z}_{\mathbf{2}}$ charge cancellation conditions, which constrain the magnetic flux quanta for the D7-brane gauge fields. We reinterpret and derive these conditions by lifting the configuration to F-/M-theory on an elliptically fibered K3, where the magnetic fields correspond to non-trivial 4-form fluxes supported on special loci (the degenerations of the elliptic fibration) in a purely geometrical background without branes. We show that proper quantization of the 4-form flux, as integer cohomology classes in K3, automatically leads to type IIB magnetic fluxes obeying the standard quantization conditions plus the additional K-theory $\mathbf{Z}_{\mathbf{2}}$ constraint. In addition, we find new $\mathbf{Z}_{\mathbf{2}}$ constraints which we properly interpret.

Moreover, we generalize the result in the presence of bulk 4 -form fluxes, leading to a modification of the $\mathbf{Z}_{\mathbf{2}}$ quantization conditions on D7-brane magnetic fluxes in the presence of non-trivial 3-form fluxes in the type IIB orientifold. Hence, our results have important consequences in the construction of consistent compactifications of type IIB theory with 3form fluxes and D-branes (see [10,11] for a general discussion of flux compactifications of this kind, and $[12,6]$ for the formalism to incorporate magnetised D-branes).

This paper is organized as follows. In Section 2 we describe the K-theory charge cancellation constraints in certain orientifold models with D-branes carrying world-volume magnetic fluxes. We also provide the lift of an orientifold with O7-planes and magnetised D7-branes in 
terms of F-/M-theory on $\mathbf{T}^{\mathbf{4}} / \mathbf{Z}_{\mathbf{2}} \times \mathbf{T}^{\mathbf{2}}$ with non-trivial 4-form fluxes along certain localized 2-forms. In Section 3 we describe the homology of $K 3$ at the $\mathbf{T}^{\mathbf{4}} / \mathbf{Z}_{\mathbf{2}}$ point, and construct a generating set of 2-cycles in terms of the structure of $\mathbf{T}^{\mathbf{4}} / \mathbf{Z}_{\mathbf{2}}$ as an elliptic fibration. In Section 4 we use this information to show that 4-form flux quantization in cohomology in F/M-theory implies the standard quantization of IIB D7-brane world-volume magnetic fluxes, plus additional discrete $\mathbf{Z}_{\mathbf{2}}$ conditions, which correspond to K-theory charge cancellation conditions. In Section 5 we show the modification of these conditions when type IIB bulk 3 -form fluxes are introduced. Finally, Section 6 contains our conclusions.

\section{K-theory constraints in magnetised D-branes}

\section{$2.1 \quad$ K-theory constraints on type I}

Consider type I string theory compactified on $\mathbf{T}^{\mathbf{2}}$, with a $\widehat{D 7}$-brane spanning the eight noncompact dimensions and sitting at a point on $\mathbf{T}^{\mathbf{2}}$. As discussed in [4], this configuration is inconsistent due to non-cancellation of the K-theory $\mathbf{Z}_{\mathbf{2}}$ charge carried by the $\widehat{D 7}$-brane. The simplest way to show the inconsistency is to introduce a D5-brane probe wrapping the $\mathbf{T}^{\mathbf{2}}$. The field theory on the $4 \mathrm{~d}$ non-compact dimensions of the probe has a gauge group $U S p(2)=S U(2)$. Due to the presence of the $\widehat{D 7}$-brane in the background, there is one Weyl fermion in the fundamental representation from the $5 \hat{7}$ open string sector. Hence the probe world-volume theory is anomalous due to a Witten global gauge anomaly [13]. This reflects an inconsistency in the background. Compactifications with an even number of $\widehat{D} 7$-branes have however proper cancellation of $\mathbf{Z}_{\mathbf{2}}$ charges and are hence consistent.

This line of argument can also show the existence of $\mathbf{Z}_{\mathbf{2}}$ constraints in type I compactifications without explicit $\widehat{D 7}$-branes, but with non-trivial world-volume gauge bundles on the D9-branes. The simplest situation is to consider a $\mathbf{T}^{\mathbf{2}}$ compactification of type I, with abelian monopole backgrounds. Namely, denote $U(1)_{a}$ the 16 Cartan generators of $S O(32)$, or equivalently, from the viewpoint of the parent IIB theory, the $U(1)$ gauge factors carried by the $a^{\text {th }}$ D9-brane and its orientifold image (denoted $a^{\prime}$ ). Consider introducing a constant $U(1)_{a}$ magnetic field $F_{a}$ on $\mathbf{T}^{2}$, satisfying the quantization condition

$$
\int_{\mathbf{T}^{2}} F_{a}=n_{a}
$$

These are the so-called magnetised D-brane constructions, studied among others in $[14,15$, $16,17]$. Notice that there is an additional freedom in choosing the multiwrapping of the D-branes on the $\mathbf{T}^{\mathbf{2}}$ [15], but for simplicity we will not include it, since it is not essential to our main point.

The magnetic field induces $n_{a}$ units of IIB D7-brane charge on the $a^{\text {th }}$ D9-brane, and of $\overline{D 7}$-brane charge on the $a^{\text {th }}$ image D9-brane. Given that a D7- $\overline{D 7}$ pair descends to a 
$\widehat{D 7}$-brane of the quotient type I theory [2], the compactification contains an overall number of $\sum_{a} n_{a}$ units of $\widehat{D 7}$-brane charge. Cancellation of the K-theory $\mathbf{Z}_{2}$ charge thus requires

$$
\sum_{a} n_{a} \in 2 \mathbf{Z}
$$

Again, the inconsistency of the backgrounds which do not satisfy the above constraint can be made manifest by introducing a D5-brane probe wrapped on $\mathbf{T}^{\mathbf{2}}$. Namely, in the 59 open string sector the index of the Dirac operator coupled to the gauge bundle is odd, leading to an odd number of $4 \mathrm{~d}$ Weyl fermions in the fundamental representation of the symplectic group on the D5-branes.

The generalization of the above discussion is straightforward. In any type I compactification on a manifold $X$, with D9-branes (and images) carrying an $S O(32)$ gauge bundle $V$, the K-theory charge corresponding to induce $\widehat{D 7}$-branes should cancel. Regarding the type I real bundle in terms of type IIB D9-branes carrying a $U(16)$ gauge bundle $E$, and their images,

cancellation of the K-theory induced $\widehat{D 7}$-brane $\mathbf{Z}_{2}$ charge requires $c_{1}(E)$ to be an even class in $H^{2}(X, \mathbf{Z})$.

\subsection{K-theory constraints on type IIB orientifolds}

Let us return to the type I $\mathbf{T}^{\mathbf{2}}$ compactification, and obtain similar conclusions for related orientifold models. Consider compactifying on a further $\mathbf{T}^{\mathbf{2}}$ (denoted $\left(\mathbf{T}^{\mathbf{2}}\right)^{\prime}$ to avoid confusion), and T-dualizing on its two directions. We obtain type IIB on $\left(\mathbf{T}^{\mathbf{2}}\right)^{\prime} \times \mathbf{T}^{\mathbf{2}}$, modded out by $\Omega R(-1)^{F_{L}}$, where $R$ flips the two coordinates of $\left(\mathbf{T}^{\mathbf{2}}\right)^{\prime}$ and leaves $\mathbf{T}^{\mathbf{2}}$ invariant. There are four O7-planes sitting at the fixed points in $\left(\mathbf{T}^{\mathbf{2}}\right)^{\prime}$, and 16 D7-branes and their images, with world-volume magnetic fields $F_{a}$ satisfying the quantization (2.1).

The above arguments show that the configuration must satisfy the additional $\mathbf{Z}_{\mathbf{2}}$ cancellation condition (2.2). By T-duality, the $\mathbf{Z}_{\mathbf{2}}$ charge is now associated to a non-BPS D5-brane ( $\widehat{D 5}$-brane) wrapped on $\left(\mathbf{T}^{2}\right)^{\prime}$. This note is devoted to developing a better understanding of the additional $\mathbf{Z}_{\mathbf{2}}$ condition in this system.

What is interesting about this configuration is that it has a direct connection with other dual descriptions in terms of compactifications of F/M-theory. Moreover, it contains several of the ingredients present in many of the recently studied flux compactifications with O3planes, D3-branes, and 7-branes $[10,11]$. In fact, the relation will be far more direct once we study the lift to F/M-theory, in the next section.

\subsection{Lift to F/M-theory with fluxes}

In this section we describe the lift of the above configurations to F/M-theory, and investigate the interpretation of the $\mathbf{Z}_{\mathbf{2}} \mathrm{K}$-theory constraint in the latter context. 
The lift of type IIB orientifolds with O7-planes and D7-branes, in the absence of worldvolume magnetic fields is familiar and has been discussed e.g. in [18]. One obtains F/M-theory ${ }^{1}$ compactified on an elliptic fibration over a base given by the IIB compactification space. Degenerations of the elliptic fibration where a $(p, q)$-cycle degenerates correspond to $(p, q)$ 7-branes in the IIB picture. As discussed in [18], the lift of O7-planes correspond to a set of two fiber degenerations, whose $(p, q)$ label can be chosen [19] to be $(1,-1)$ and $(3,-1)$, in the convention where a D7-brane lifts to a $(1,0)$ degeneration.

The world-volume gauge field on the 7-branes arises as follows. The local geometry around the pinching point of a degenerate elliptic fiber is that of a Taub-NUT geometry. This geometry supports a normalizable harmonic 2-form [20], which we denote by $\omega$. In the M-theory picture, the Kaluza-Klein reduction of the 3-form leads to a component of the type

$$
C_{3}=\omega \wedge A_{1}
$$

where $A_{1}$ is a 1 -form supported on the degeneration locus on the base, namely on the 7-brane world-volume. Hence, it corresponds to the gauge field carried by the 7-brane. Therefore, the world-volume magnetic fluxes on a 7-brane correspond to components of the 4 -form field strength, of the type

$$
G_{4}=\omega \wedge F_{2}
$$

Namely, they are mapped to standard supergravity 4-form fluxes in an otherwise purely geometrical compactification. Hence, interestingly the question of the constraints (quantization and additional $\mathbf{Z}_{\mathbf{2}}$ condition) on the IIB 7-brane magnetic fluxes is intimately related to the quantization conditions of 4 -form fluxes in F/M-theory compactifications.

The setup corresponds to compactifications of F/M-theory with non-trivial $G_{4}$ field strength fluxes, which have been under intense study by themselves on in their type IIB avatar $[21,10,11]$. For the moment we simply consider $G_{4}$ fluxes which receive the interpretation of IIB gauge backgrounds, leaving the discussion of $G_{4}$ fluxes which reduce to IIB 3-form fluxes for Section 5 .

One may wonder if quantization of $G_{4}$ as an integer cohomology class is sufficient to guarantee the consistency of the backgrounds, or if some additional discrete constraint is required in order to account for the extra $\mathbf{Z}_{\mathbf{2}}$ condition in string theory. In coming sections we study a particular compactification on K3, which is simple enough for detailed analysis, but still keeps all the required subtle structure. We show that standard integer quantization of $G_{4}$ automatically implies the appropriate constraints on the IIB 7-brane world-volume magnetic fluxes, including the above mentioned $\mathbf{Z}_{2}$ constraint.

\footnotetext{
${ }^{1}$ The relation to M-theory arises upon an additional compactification on a circle. Since the background (geometry plus fluxes) is most physical in the M-theory setup, we center on this picture when describing it. Note however that the M-theory backgrounds surviving in the F-theory limit are not the most general ones. We use the notation F/M-theory to refer to M-theory on backgrounds of the former kind.
} 


\subsection{Gauge fields for $\mathrm{K} 3$ at the $S O(8)^{4}$ point}

In an F-theory compactification, components of the 3-form along 2-forms localized at pinchings of the elliptic fiber correspond to gauge fields on the corresponding IIB 7-branes. However, not all such localized 2-forms are independent, and the total number of gauge fields is smaller than the number of degenerate fibers [22]. Our strategy in the present paper is to pick a set of basic degenerations, which generate all gauge fields in the theory, so that one can safely ignore the 2-forms supported on the remaining degenerations (which can be regarded as non-dynamical).

This splitting is manifestly natural and justified in F/M-theory models which correspond to lifts of type IIB orientifold with local cancellation of 7-brane charge, namely with sets of exactly four D7-branes on top of each O7-plane. In the corresponding lift, we locally find a Kodaira $I_{0}^{*}$ degenerate fiber [18], to be discussed later on. Slightly separating the D7branes from the orientifold plane leads to a lift with four $(1,0)$ fibers, corresponding to the D7-branes, and a set of $(1,-1)$ and $(3,-1)$ fibers, corresponding to the O7-plane. In this setup is it natural to associate the independent 2 -forms $\omega_{i}$ of the $(1,0)$ degenerations to the D7-branes, while the 2-forms of the remaining two fibers are not independent, and do not generate new gauge fields (as corresponds to their interpretation as an O7-plane). Far away from such situation, the relation between the gauge fields in the lower-dimensional theory and the localized 2-forms is more involved, and we will not enter its discussion in the present note.

The simplest situation of the above kind corresponds to the setup in Section 2.2, namely type IIB on $\left(\mathbf{T}^{\mathbf{2}}\right)^{\prime}$ modded out by $\Omega R$, with 8 D7-branes (four and their four images) on top of each of the four O7-planes. The gauge symmetry from the D7-branes corresponds to $S O(8)^{4}$. The F-theory lift corresponds to a compactification on the $\mathbf{T}^{\mathbf{4}} / \mathbf{Z}_{\mathbf{2}}$, orbifold limit of K3. The appearance of the gauge symmetry in this setup is described below. We refer to this locus in the moduli space of the system as the $S O(8)^{4}$ point.

Following the above general strategy, the F/M-theory lift of the Cartan gauge fields on the $16 \mathrm{D} 7$-branes at the $S O(8)^{4}$ point, and its corresponding field strengths, correspond to supergravity backgrounds for the M-theory 3 -form $C_{3}$ and its field strength $G_{4}$

$$
C_{3}=\sum_{a=1}^{16} \omega_{a} \wedge A_{1}^{(a)} \quad, \quad G_{4}=\sum_{a=1}^{16} \omega_{a} \wedge F_{2}^{(a)}
$$

where $\omega_{a}$ label the 2-forms supported on the degenerations corresponding to D7-branes in the IIB reduction. Clearly, the 2-forms associated to other degenerate fibers do not lead to dynamical fields in the IIB reduction, hence showing they are not independent degrees of freedom. 


\section{Geometry of K3 at the $S O(8)^{4}$ point}

\subsection{The homology lattice of $\mathrm{T}^{4} / \mathrm{Z}_{2}$}

A general discussion of the homology lattice of K3 may be found in [23]. Here, as mentioned, we consider for concreteness the $\mathrm{K} 3$ at the $S O(8)^{4}$ point, to make the connection with the type IIB orientifolds more manifest [18]. Hence we consider the K3 to be given by the orbifold $\mathbf{T}^{4} / \mathbf{Z}_{2}$, which for simplicity we also take to be rectangular.

A basis of $H_{2}\left(\mathbf{T}^{\mathbf{4}} / \mathbf{Z}_{\mathbf{2}}, \mathbf{R}\right)$, which is 22-dimensional, is provided by the following set of 2-cycles: We first have the six independent 2-cycles on $\mathbf{T}^{\mathbf{4}}$, which are invariant under the $\mathbf{Z}_{\mathbf{2}}$ action. We also have the 16 two-spheres arising from blowing up the 16 local $\mathbf{C}^{2} / \mathbf{Z}_{\mathbf{2}}$ orbifold singularities.

It is convenient to introduce some notation for these 2-cycles. Let us label the four coordinates in $\mathbf{T}^{\mathbf{4}}$ by latin indices $i=1,2,3,4$. We denote $\Pi_{i j}$ (antisymmetric in the two indices) the toroidal 2-cycle spanning the directions $i, j$. The sixteen blowup 2-cycles sit at points with coordinates 0 or $1 / 2$ along each of the four coordinates (in units of the corresponding radius). Denoting these two possible values by + and - , we denote the blowup 2-cycles by $e_{a_{1} a_{2} a_{3} a_{4}}$, where $a_{i}=+,-$ for $i=1,2,3,4$ denotes the location of the $\mathbf{Z}_{\mathbf{2}}$ fixed point in the $i^{\text {th }}$ direction in $\mathbf{T}^{\mathbf{4}}$.

It is easy to realize that the above set of 2-cycles, which span the so-called Kummer lattice, do not form the complete lattice of the K3 second homology. Rather, they form a sublattice of index two (see e.g. [23, 24] for discussion). Indeed, there exist 2-cycles in the quotient, spanning two directions of the $\mathbf{T}^{\mathbf{4}}$ and passing through four $\mathbf{Z}_{\mathbf{2}}$ fixed points, which have half the volume of the corresponding toroidal cycles. Since these 2-cycles are mapped to themselves by the $\mathbf{Z}_{\mathbf{2}}$ action, one may think about them as fractional 2-cycles ${ }^{2}$. It is convenient to introduce some notation for these 2-cycles, as follows. Consider e.g. the 2cycle spanning the directions 3,4 , and passing through the fixed points associated to $e_{++++}$, $e_{+++-}, e_{++-+}$and $e_{++--}$. We denote its class by $f_{++34}$. Clearly $f_{++34}$ and $\Pi_{34}$ are not independent, but satisfy the relation

$$
2 f_{++34}+e_{++++}+e_{+++-}+e_{++-+}+e_{++--}=\Pi_{34}
$$

Clearly, there exist 2-cycles $f_{+-34}, f_{-+34}$ and $f_{--34}$ spanning the directions 3,4 but passing through a different set of fixed points, and having a similar relation to $\Pi_{34}$. Also, there are classes $f$ associated to any other pair of directions. A more explicit realization of the 2-cycles $f$ will be manifest in coming paragraphs.

\footnotetext{
${ }^{2}$ This is convenient in that a brane wrapped on such 2-cycle is described as a fractional brane in orbifold language. In the geometric sense it is somewhat of a misnomer, since it belongs to the K3 integer homology lattice.
} 

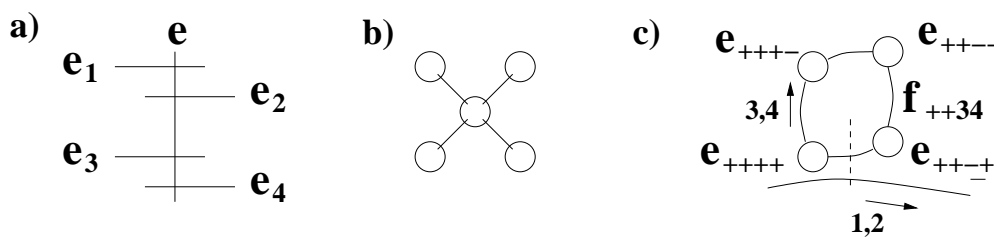

Figure 1: a) The non-trivial 2-cycles in a Kodaira $I_{0}^{*}$ singular fiber b) the Dynkin diagram of $S O(8)$ c) realization over a degeneration point in $\mathbf{T}^{\mathbf{4}} / \mathbf{Z}_{\mathbf{2}}$.

The intersection numbers of these cycles can be obtained from

$$
\begin{aligned}
\Pi_{i j} \cdot \Pi_{k l} & =\epsilon_{i j k l} \\
\Pi_{i j} \cdot e_{a_{1} a_{2} a_{3} a_{4}} & =0 \\
e_{a_{1} a_{2} a_{3} a_{4}} \cdot e_{b_{1} b_{2} b_{3} b_{4}} & =-2 \delta_{a_{1} b_{1}} \delta_{a_{2} b_{2}} \delta_{a_{3} b_{3}} \delta_{a_{4} b_{4}}
\end{aligned}
$$

and relations similar to (3.1). For instance we have $f_{++34} \cdot e_{++a_{3} a_{4}}=1$, etc.

\subsection{Homology of $\mathrm{T}^{4} / \mathrm{Z}_{2}$ as en elliptic fibration}

In order to connect with the type IIB orientifold model, it is convenient to describe the homology lattice of this K3 in terms of its interpretation as an elliptic fibration. Consider the torus spanned by the coordinates 34 (namely $\Pi_{34}$ ) to be the elliptic fiber. The fibration has four degenerate fibers, located over the points of the base $\mathbf{T}^{\mathbf{2}}$ fixed by the $\mathbf{Z}_{\mathbf{2}}$ action. As described in [18], the degenerate fibers are of Kodaira type $I_{0}^{*}$, and lead, in the F-theory limit of shrinking fiber class, to an enhanced $S O(8)$ gauge symmetry. Indeed (see e.g. [23]) the (extended) Dynkin diagram of this symmetry is realized in terms of the 2-cycles of the reducible $I_{0}^{*}$ fiber, as shown in figures 1a,b. This diagram encodes the intersection numbers among the classes, namely

$$
e \cdot e=-2 \quad, \quad e_{\alpha} \cdot e_{\beta}=-2 \delta_{\alpha \beta} \quad, \quad e \cdot e_{\alpha}=1
$$

The 2-cycles are related to the fiber class by

$$
2 e+e_{1}+e_{2}+e_{3}+e_{4}=f
$$

The relation of these 2-cycles with the above general description is clear. Let us center on a particular degeneration point on the base, e.g. that corresponding to the origin in the coordinates 1,2 . Using our general description above, on top of this point we have the class $f_{++34}$, and the blowup classes $e_{++a b}$. Their intersection numbers, along with the relation (3.1), leads to the relation

$$
f_{++34}=e \quad, \quad e_{1}=e_{++++} \quad, \quad e_{2}=e_{+++-} \quad, \quad e_{3}=e_{++-+} \quad, \quad e_{4}=e_{++--}
$$


as illustrated in figure 1c. The above relation is unique up to the action of the Weyl group, and we will use the above convention in the remainder of the paper. Namely, $\alpha$ is a double index $\left(a_{3}, a_{4}\right)$ denoting the position on the fiber, with $\alpha=1,2,3,4$ corresponding to $a_{3} a_{4}=$ ,,,+++--+-- .

The above discussion shows that the class $e$ is precisely one of the classes required to refine the Kummer lattice to the actual integer homology lattice of K3. Similar discussions apply to the classes $f_{a_{1} a_{2} 34}$.

It is also possible to provide a description of the remaining classes $f$ in terms of the elliptic fibration picture. The simplest are the classes $f_{12 a_{3} a_{4}}$, which correspond to different sections of the torus fibration ${ }^{3}$. The remaining classes e.g. $f_{1 a_{2} 3 a_{4}}$ are related to the 2 -torus classes $\Pi_{13}$, etc. In the elliptic fibration, 2 -cycles like $\Pi_{13}$ are realized by considering a circle on the base, spanning the direction 1, and fibering over it the circle of the fiber corresponding to 3. An important point is that the circle on the base surrounds two $I_{0}^{*}$ degenerations, each of them leading to an $S L(2, \mathbf{Z})$ monodromy $-\mathbf{1}_{2}$, so the total monodromy along the base circle is trivial, and the circle along 3 can be consistently fibered to form a 2-cycle. Actually, this representative of $\Pi_{13}$ can be moved toward the two $I_{0}^{*}$ degeneration points (corresponding e.g. to the coordinate positions $(++)$ and $(-+)$ on the base), where it becomes reducible via

$$
\Pi_{13}=2 f_{1+3+}+e_{++++}+e_{++-+}+e_{-+++}+e_{-+-+}
$$

Namely, the class $f_{1+3+}$ corresponds to a 2-cycle spanning the directions 1,3 , located at origin in the direction 2,4 , and passing through the above four fixed points. Clearly one can operate similarly to describe the remaining classes of type $f$.

\section{Type IIB gauge field flux quantization from $\mathrm{F} / \mathrm{M}$-theory}

In this section we employ the quantization conditions on 4-form fluxes in F/M-theory to obtain the corresponding quantization conditions for the IIB gauge field fluxes. The key point in the analysis is the computation of the periods of the 2 -forms $\omega$ associated to the D7branes. The bottom line is that these 2 -forms are not integer cohomology classes, but suitable linear combinations of them are. As we show this implies that appropriate quantization of $G_{4}$ imposes the extra constraint on the D7-brane gauge field fluxes (2.2), as well as some previously unnoticed ones.

\subsection{The forms $\omega$ revisited}

In this section we determine the integrals of the forms $\omega$, involved in the definition of the D7-brane gauge fields, over different 2-cycles in the homology lattice of K3. A simple way

\footnotetext{
${ }^{3}$ Since an elliptic curve is a torus with a choice of a point, the elliptic fibration implies the choice of one of these sections as the base.
} 
a)

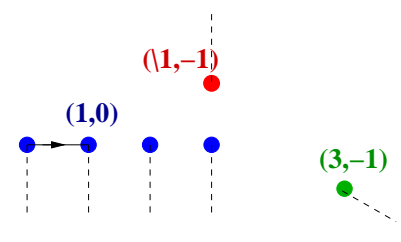

b)

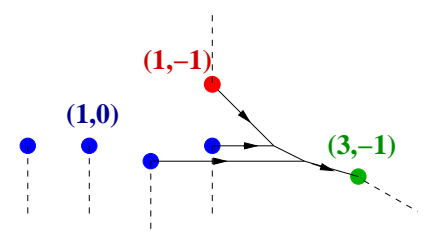

Figure 2: Some junctions representing BPS states near a Kodaira $I_{0}^{*}$ singularity. Labels denote the $(p, q)$ type of degeneration of points of the corresponding color, with the blue $(1,0)$ nodes corresponding to D7-branes in the IIB interpretation. Figure a) represents the state corresponding to the $S O(8)$ nonzero roots of the form $(+1,-1,0,0)$. Figure b) represents the state corresponding to the non-zero root $(0,0,+1,+1)$. For clarity, one of the D7-branes has been slightly shifted downward. The remaining non-zero roots are obtained by choosing similar junctions involving different pairs of D7-branes.

to obtain these integrals is to compute the charges of BPS states (corresponding to branes wrapped on such 2-cycles) under the Cartan generators of the associated $S O(8)$ gauge symmetries.

\section{Cycles localized at $I_{0}^{*}$ fibers}

The realization of the homology classes of $\mathbf{T}^{\mathbf{4}} / \mathbf{Z}_{\mathbf{2}}$ from the elliptic fibration viewpoint makes the relation to the type IIB orientifold manifest. In particular, it is possible to realize the 2-cycles associated to the above classes $e, e_{i}$ in an $I_{0}^{*}$ fiber in terms of string junctions [25] in the IIB orientifold. Hence junction techniques allow to compute the integrals of the 2-forms $\omega_{i}$ over those 2-cycles. Physically this amounts to computing the charges of the BPS states corresponding to M-theory M2-branes wrapped on those 2-cycles. In junction language, they are simply given by the numbers of string prongs ending on the relevant D7-branes, counted with orientation.

The junctions for the 2-cycles of type $e, e_{i}$, are shown in figure 2 , and it can be checked that their intersection numbers (determined using junction rules) agree with (3.3). The charges of the corresponding BPS states with respect to the four $S O(8)$ Cartan generators, or equivalently the integrals of the different 2 -forms $\omega_{i}$ over a given 2 -cycle, can be described by a vector

$e_{1}=(1,-1,0,0), e_{2}=(1,1,0,0), e_{3}=(0,0,1,-1), e_{4}=(0,0,1,1), e=(-1,0,-1,0)$

The $S O(8)$-root structure of these vectors is manifest.

\section{Additional 2-cycles}

In the previous discussion we have determined the integrals of the forms $\omega_{i}$ associated to a given $S O(8)$ along the 2-cycles localized on that degenerate fiber. However, it is important to know the integrals over other 2-cycles in the K3. As usual, this can be done by wrapping M2-branes on the corresponding M-theory 2-cycles and computing the charges of the 
corresponding states under the Cartan generators of the $S O(8)$.

Let us consider a IIB D1-brane passing through two stacks of O7/D7-branes, e.g. with coordinates ++ and -+ in the directions 1,2 (being fixed under the orientifold action, this is a half D1-brane). The charges of this state under the Cartan generators of the two $S O(8)$ 's through which it passes are easily computed in the IIB picture. In the sector of 1-7 open strings, there are fermion zero modes in the vector representation of the $S O(8)$ 's. Upon quantization of these zero modes, the object transforms as a spinor simultaneously under both $S O(8)$ 's. The overall chirality is fixed by the GSO projection, so the state transforms in the $\left(8_{s}, 8_{s}\right)+\left(8_{c}, 8_{c}\right)$. Namely, the weight vector can be written

$$
\left( \pm \frac{1}{2}, \pm \frac{1}{2}, \pm \frac{1}{2}, \pm \frac{1}{2} ; \pm \frac{1}{2}, \pm \frac{1}{2}, \pm \frac{1}{2}, \pm \frac{1}{2}\right)
$$

with a total even number of minus signs. This gives the charge of the (multiplet of) states under the Cartan generators of the two $S O(8)$ 's. Notice that different states in a multiplet are related by addition of vectors of type (4.1).

The above set of charges can be understood from the geometric viewpoint as follows. From the F/M-theory viewpoint the above D1-brane state corresponds to an M2-brane wrapped on a cycle spanning the directions 1,3 , for instance $f_{1+3+}$. From the intersection numbers with the exceptional classes $e_{++a_{3} a_{4}}$ and $e_{-+a_{3} a_{4}}$, and the expression of the latter in terms of vectors (4.1), it follows that an M2-brane on $f_{1+3+}$ has a charge vector

$$
\left(-\frac{1}{2}, \frac{1}{2},-\frac{1}{2}, \frac{1}{2} ;-\frac{1}{2}, \frac{1}{2},-\frac{1}{2}, \frac{1}{2}\right)
$$

under the Cartan generators of the $S O(8)$ factors located at $\left(a_{1}, a_{2}\right)=(+,+),(-,+)$. By considering M2-brane on other holomorphic 2-cycles, differing from $f_{1+3+}$ or $f_{1+3}$ - by the addition of exceptional classes, one can generate the whole multiplet ${ }^{4}$ of states (4.2).

This implies that the integrals of the forms $\omega_{i}$ of the corresponding $I_{0}^{*}$ degenerations are half-integers. Notice again that this does not imply any contradiction with the integer intersection numbers between 2-cycles in the integer lattice of K3. Clearly, similar states can be constructed for any pair of $S O(8)$ factors, leading to bi-spinors of the corresponding $S O(8)^{2}$ groups.

Finally, we can also consider fundamental strings stretching through pairs of $S O(8)$ groups. This leads to states in the representation $\left(8_{v}, 8_{v}\right)$ under them. In the M-theory realization, we have M2-branes wrapped on e.g. $f_{1++4}$. The intersection numbers of this class with $e_{++a_{3} a_{4}}$ and $e_{-+a_{3} a_{4}}$ lead to a set of charges

$$
(-1,0,0,0 ;-1,0,0,0)
$$

Again, the rest of the multiplet may be obtained by adding exceptional classes. Hence the integrals of the forms $\omega_{i}$ over those cycles are integers.

\footnotetext{
${ }^{4}$ Actually, in order to generate the whole multiplet, we need to consider the addition of e.g. the class $f_{1++4}$ mentioned below. This simply means considering D1-branes with some induced F1 charge.
} 


\subsection{Quantization conditions}

The above characterization of the forms $\omega_{i}$ is sufficient to understand the relation between flux quantization in F/M-theory and string theory, as we do in this section.

Start with a set of D7-brane magnetic fields $F_{A, i}$, where $A$ labels the $S O(8)$ factor, and can be regarded as a double index $A=\left(a_{1}, a_{2}\right)$ encoding the coordinates in the directions 1,2. This configuration can be lifted to F/M-theory as

$$
G_{4}=\sum_{A} \sum_{i} \omega_{A, i} \wedge F_{A, i}
$$

The requirement that the flux $G_{4}$ is quantized over 4-cycles of the kind $e_{A} \times \mathbf{T}^{\mathbf{2}}$, where $e_{A}$ is an exceptional class over the $A^{\text {th }} I_{0}^{*}$ fiber, implies that

$$
\int_{\mathbf{T}^{\mathbf{2}}} F_{A, i}=n_{A, i} \in \mathbf{Z}
$$

which is the familiar quantization of D-brane world-volume magnetic fields. In addition, we have the conditions that the flux $G_{4}$ is quantized over 4 -cycles of the kind $f \times \mathbf{T}^{2}$, where $f$ is a 2 -cycle passing through the elliptic degeneration $A$ and $B$ on the base. For instance, $f_{1+3+}$ for $A=(++), B=(-+)$. These conditions imply

$$
\sum_{i}\left(n_{A, i}+n_{B, i}\right) \in 2 \mathbf{Z}
$$

This should hold for any pair $A, B$. Clearly by combining these conditions one can obtain the constraint

$$
\sum_{i, A} n_{A, i} \in 2 \mathbf{Z}
$$

where the sum clearly runs over all $U(1)$ magnetic fields in the theory. This is the condition (2.2), which arises from K-theory in the IIB language. In our setup, it has automatically arisen from proper quantization of fluxes in F/M-theory. In other words, the $\omega$ 's are not integer cohomology classes, but suitable linear combinations of them are. The requirement that $G_{4}$ is an integer cohomology class forces the magnetic flux quanta to adjust so that one recovers linear combinations of $\omega$ 's which are integer classes.

This result nicely agrees with the observation in [26] that K-theory in string theory arises from homology in M-theory.

\subsection{New constraints on gauge field fluxes}

The conditions (4.7) imply (4.8), but are actually stronger. We conclude this section with a discussion of these new $\mathbf{Z}_{\mathbf{2}}$ conditions on the quantization gauge field fluxes arising the 4-form flux quantization. 
In type IIB string theory, these conditions do not correspond to cancellation of a cohomological charge, hence they should correspond to cancellation of a K-theory charge. Indeed, let us consider making $\mathbf{T}^{\mathbf{2}}$ non-compact and studying the structure of the world-volume gauge bundle over the resulting non-compact 2-plane. In this setup, the quantity

$$
\sum_{i=1}^{4} \int_{\mathbf{R}^{2}} F_{A, i}+\sum_{i=1}^{4} \int_{\mathbf{R}^{2}} F_{B, i}
$$

mod 2 corresponds to a non-trivial $\mathbf{Z}_{\mathbf{2}}$ charge carried by the bundles. This $\mathbf{Z}_{\mathbf{2}}$ charge can be measured by considering the particle obtained by wrapping a D1-brane on the fixed circle passing through the $A^{\text {th }}$ and $B^{\text {th }}$ fixed points (hence transforming as a spinor under both $S O(8)$ 's), and moving it around the circle at infinity in the 2-plane. The condition (4.7) simply amounts to requiring cancellation of this K-theory charge in the compact context.

Notice the intricate relation between the existence of the non-trivial $\mathbf{Z}_{\mathbf{2}}$ charge and the global structure of the gauge group (which precisely allows the existence of the above bispinor state). Hence the above statement depends crucially on the global structure of the gauge group, which can be described as follows. Define the $\operatorname{Spin}(8)^{4}$ element $x_{A B}$ as that acting as -1 on the representation $\left(8_{v}, 1\right)+\left(1,8_{v}\right)$ and $\left(8_{s}, 8_{c}\right)$ and $\left(8_{c}, 8_{s}\right)$ of the $A^{\text {th }}$ and $B^{\text {th }} \operatorname{Spin}(8)$ factors, and as +1 on $\left(8_{s}, 8_{s}\right),\left(8_{c}, 8_{c}\right)$ and the adjoint representations. The set of $x_{A B}$ 's form a subgroup $\Gamma$ of $\operatorname{Spin}(8)^{4}$. The gauge group of the our compactification is globally $\operatorname{Spin}(8)^{4} / \Gamma$, so that there is an obstruction to define states in the representations picking minus signs under these actions ${ }^{5}$, and such states are indeed absent from the theory. On the other hand, states transforming in the $\left(8_{s}, 8_{s}\right)$ or $\left(8_{c}, 8_{c}\right)$ can be (and are) present, and can be used to measure the $\mathbf{Z}_{\mathbf{2}}$ charge carried by the bundles. Hence, the classification of K-theory classes of bundles with this global group structure should contain the $\mathbf{Z}_{\mathbf{2}}$ charges encountered above. In a compact setup, consistency requires the cancellation of such $\mathbf{Z}_{\mathbf{2}}$ charges.

Finally, let us mention that the global structure of the gauge group (and hence the associated K-theory) makes it clear from the string viewpoint that there are no additional discrete constraints. Namely, ignoring the global structure of the gauge group one might have been tempted to interpret the quantity $\sum_{i} n_{A, i} \bmod 2$ as a discrete charge carried by each $S O(8)$ factor, which should therefore be canceled in a compact setup. However, the only way to detect this quantity as a physical charge would be to probe the theory with a state in the spinor of just one of the $\operatorname{Spin}(8)$ factors. Such states are not present in the theory (and in fact are not allowed by the global structure of the group), hence the above quantity is not a physical charge of the system, and we need not require its cancellation, even in a compact setup.

\footnotetext{
${ }^{5}$ The obstruction to define such states could be defined as the generalized Stiefel-Whitney class in [27] obstructing vector structures in the different $\operatorname{Spin}(16)$ 's containing $\operatorname{Spin}(8)_{A} \times \operatorname{Spin}(8)_{B}$.
} 


\section{Adding 3-form fluxes}

The lift of world-volume magnetic fluxes on D7-branes naturally leads to M-theory compactifications with non-trivial $G_{4}$-flux along the localized forms $\omega$. It is therefore natural to discuss the situation with more general $G_{4}$-fluxes. As discussed in $[10,11]$, the only additional components of $G_{4}$ which survive in the F-theory limit are those with one leg in the elliptic fiber, and correspond to type IIB NSNS and RR 3-form fluxes, $H_{3}$ and $F_{3}$ respectively. Defining the complex flux $G_{3}=F_{3}-\tau H_{3}$, where $\tau$ is the IIB complex coupling (complex structure of the fiber torus), we have the relation

$$
G_{4}=\frac{G_{3} d \bar{w}}{\bar{\tau}-\tau}+\text { h.c. }
$$

where $d w=d x_{4}+i d x_{3}$. In our situation of square torus, $\tau=i$ and

$$
G_{4}=H_{3} d x_{4}+F_{3} d x_{3}
$$

Hence a general configuration of $G_{4}$-flux corresponds to a general configuration of type IIB $G_{3}$-flux and D7-brane world-volume magnetic fluxes.

Given that world-volume and 3-form fluxes in IIB are components of the same kind of flux in F/M-theory, it is important to consider the possible effect of bulk 3-form fluxes on the quantization conditions for world-volume magnetic fluxes. In this section we address this issue and find that the conditions are modified.

Clearly, the only components of $G_{3}$ which may have a non-trivial effect of the D7-brane magnetic quanta $n_{a}$ are those with one leg on $\left(\mathbf{T}^{\mathbf{2}}\right)^{\prime}$ and two on $\mathbf{T}^{\mathbf{2}}$. Namely

$$
H_{3}=N_{14} d x_{1} d x d y+N_{24} d x_{2} d x d y \quad, \quad F_{3}=N_{13} d x_{1} d x d y+N_{23} d x_{2} d x d y
$$

where $d x d y$ is the volume form of $\mathbf{T}^{\mathbf{2}}$, and the coefficient notation and signs are set for convenience. The coefficients $N$ is this expression denote the quanta of 3 -form flux along toroidal cycles. Since the orientifold action actually quotients $\left(\mathbf{T}^{\mathbf{2}}\right)^{\prime}$ by a $\mathbf{Z}_{\mathbf{2}}$ action, one might think that the flux quanta are forced to be even. However, as we show using the F/M-theory picture, the quantization conditions are trickier and allow for odd quanta in certain situations. The cases including odd quanta are however more subtle and lead to a modification of the quantization conditions (4.7) for 2-form magnetic field fluxes.

In the F/M-theory picture we can parametrize the relevant $G_{4}$ schematically as

$$
G_{4}=\left(N_{13} d x_{1} d x_{3}+N_{23} d x_{2} d x_{3}+N_{14} d x_{1} d x_{4}+N_{24} d x_{2} d x_{4}+\sum_{a=1}^{16} n_{a} \omega_{a}\right) \wedge d x d y
$$

The key point is that forms like $d x_{1} d x_{3}$ are not integer classes in K3, since their integrals along 2-cycles like $f_{1+3+}$ measure the volume of the latter, and are half-integers, since the volume is half that of a toroidal cycle. However, although forms like $d x_{1} d x_{3}$ and $w_{a}$ are not 
integer classes, suitable linear combinations of them are. Hence, quantization of $G_{4}$ implies that the coefficients in the above expression must adjust to form such linear combinations.

In the following we center on a particular set of fluxes, which suffices to illustrate the main point. Consider

$$
G_{4}=\left(N_{13} d x_{1} d x_{3}+\sum_{A, i} n_{A, i} \omega_{A, i}\right) \wedge d x d y
$$

Since e.g. $\int_{f_{1+3+}} d x_{1} d x_{3}=1 / 2$, requiring this flux to be quantized over the 4 -cycle $f_{1+3+} \times \mathbf{T}^{\mathbf{2}}$ leads to the condition

$$
N_{13}+\sum_{a 3, a 4}\left(n_{++a_{3} a_{4}}+n_{-+a_{3} a_{4}}\right) \in 2 \mathbf{Z}
$$

Hence for even $N_{13}$ we recover the condition (4.7), while for odd $N_{13}$ we find a modified quantization condition on the D7-brane world-volume magnetic fluxes. Notice that the quantization condition (4.8) is unchanged.

The general pattern is clear. Given a pair of $S O(8)$ gauge factors, the sum of the magnetic flux quanta for their Cartan generators must be even (resp. odd) if the number of RR 3-form flux quanta along the corresponding 1-cycle (times $\mathbf{T}^{\mathbf{2}}$ ) is even (resp. odd) ${ }^{6}$.

On the other hand, one can use similar computations to show that NSNS fluxes do not modify the world-volume magnetic flux quantization conditions. This follows from the fact that the integrals of the forms $\omega_{A, i}$ over 2 -cycles like $f_{1++4}$ are integer. This also implies that and odd quantum of NSNS flux cannot be compensated by a suitable choice of magnetic flux quanta. Therefore, proper quantization of the M-theory 4-form flux leads to IIB NSNS fluxes quantized to even integers. The asymmetry in the behaviour of NSNS and RR fluxes may appear striking, but one should recall that the IIB background at hand contains D7-branes, hence is not invariant under S-duality.

The modification of the world-volume flux quantization conditions shows that the presence of 3-form fluxes modifies the K-theory of the configuration. It would be highly desirable to develop a better understanding of this, possible in terms of some twisted K-theory [2]. However, the modification we have found is due to RR 3-form fluxes, while twisted K-theory has been suggested as the right formalism to incorporate NSNS 3-form fluxes. We leave this as an open question.

Finally recalling our discussion in Section 4.3, the change in the quantization conditions reflects a change in the global structure of the gauge group. This is the first example of such an effect, as far as we are aware of. Again, it would be interesting to gain a better IIB interpretation of this effect.

\footnotetext{
${ }^{6}$ This kind of condition is similar of that encountered in toroidal compactifications with O3-planes, with odd 3-form flux quanta $[28,29]$. It would be interesting to understand a possible relation between the two mechanisms.
} 


\section{Final comments}

In this paper we have considered the F/M-theory lift of configurations of D7-branes with world-volume magnetic fluxes, and described the appearance of the quantization conditions on the latter (involving the additional subtle K-theory constraints) from quantization of 4form fluxes as integer cohomology forms in the former.

This result fits nicely with the computation in [26] where the partition function of Mtheory on $X_{10} \times \mathbf{S}^{\mathbf{1}}$ with fluxes classified by cohomology classes reproduced the partition function of type IIA string theory on $X_{10}$ with fluxes classified by K-theory. Our result is admittedly more modest since it has been derived just in a particular example; it would clearly be nice to develop a more general description. However, it also incorporates additional subtleties since the matching involves both bulk and localized fluxes. In this sense our matching suggests a generalization of results in the line of [26] to situations with degenerate fibers in the M-theory geometry.

We have moreover extended our analysis to the situation with non-trivial type IIB 3-form fluxes, and shown there is a non-trivial interplay between quantization of bulk 3-form fluxes and world-volume magnetic fluxes. This manifests the rich interplay between fluxes and branes, and shows that it is in general non-trivial to combine D-brane configurations with bulk flux backgrounds. Extreme care must thus be applied to exploit the modular structure of the theory in model building.

We would like to conclude with some additional comments:

- In the type IIB language, the K-theory constraints on the quantization conditions can be ultimately understood in terms of the global structure of the gauge group, namely in terms of the full set of gauge representations present in the spectrum of the theory. In this sense, the quantization conditions are more manifest in the F/M-theory picture, where states in a sufficient number of different representations are all realized on an equal footing, in terms of M2-branes wrapped on holomorphic 2-cycles. A similar comment applies to the heterotic dual of our model, where all these states are manifest in the perturbative spectrum as vectors in the Narain lattice. Hence, in heterotic theory, a straightforward analysis shows that there are no extra conditions beyond the quantization conditions following from the perturbative spectrum of the theory.

- Our analysis has shown an equivalence between cancellation of (discrete K-theory) RR charges and proper quantization of $p$-form field strength fluxes. It is interesting that these two kinds of consistency conditions, which are often thought of as independent, are ultimately related. In retrospect, such relation is not unnatural, since both kinds of conditions are related to cancellation of global anomalies.

- Quantization conditions can be regarded as arising from the requirement of absence of 
global anomalies (namely in the definition of the path integral of a suitably charged state). An amusing feature of the extra K-theory condition (2.2) is that its violation leads to a very familiar global anomaly, namely a Witten $S U(2)$ global anomaly, on the world-volume of a suitable probe, a D7-brane wrapped on $\mathbf{T}^{\mathbf{4}}$. In the F/M-theory picture, this implies that, in this particular case, incorrectly quantized 4-form fluxes can be detected as Witten anomalies on suitable probes. It would be interesting to find other setups where flux quantization is related to such a familiar global anomaly.

Our main tool in deriving our results has been the extension of known string dualities to situations with non-trivial field strength fluxes. We expect many new interesting and surprising relations from such extensions.

\section{Acknowledgements}

We thank F. Marchesano for useful conversations. A.M.U. thanks M. González for kind encouragement and support. I.G.E thanks the CERN theory unit for hospitality during completion of this work. This work has been partially supported by CICYT (Spain), under project FPA-2003-02877, and the RTN networks MRTN-CT-2004-503369 'The Quest For Unification: Theory Confronts Experiment', and MRTN-CT-2004-005104 'Constituents, Fundamental Forces and Symmetries of the Universe'. The work by I.G.E. is supported by the Gobierno Vasco PhD fellowship program.

\section{References}

[1] R. Minasian, G. W. Moore, 'K theory and Ramond-Ramond charge', JHEP 9711 (1997) 002, hep-th/9710230.

[2] E. Witten, 'D-branes and K theory', JHEP 9812 (1998) 019, hep-th/9810188.

[3] A. Sen, 'NonBPS states and Branes in string theory', in Cargese 1999, 'Progress in string theory and M-theory', 187-234 and in 'Santiago de Compostela 1999, Supersymmetry in the theories of fields, strings and branes' 45-9, hep-th/9904207.

[4] A. M. Uranga, 'D-brane probes, RR tadpole cancellation and K theory charge', Nucl. Phys. B598 (2001) 225, hep-th/0011048.

[5] F. Marchesano, 'Intersecting D-brane models', Ph.D. Thesis, hep-th/0307252.

[6] J. F.G. Cascales, A. M. Uranga, 'Chiral 4d string vacua with D branes and NSNS and RR fluxes', JHEP 0305 (2003) 011, hep-th/0303024.

[7] F. Marchesano, G. Shiu, 'MSSM vacua from flux compactifications', Phys. Rev. D71 (2005) 011701, hep-th/0408059; 'Building MSSM flux vacua', JHEP 0411 (2004) 041, hep-th/0409132. 
[8] B. Gato-Rivera, A.N. Schellekens, 'Remarks on Global Anomalies in RCFT Orientifolds', hep-th/0510074.

[9] G. W. Moore, E. Witten, 'Selfduality, Ramond-Ramond fields, and K theory', JHEP 0005 (2000) 032, hep-th/9912279; D. S. Freed, M. J. Hopkins, 'On Ramond-Ramond fields and K theory', JHEP 0005 (2000) 044, hep-th/0002027.

[10] K. Dasgupta, G. Rajesh, S. Sethi, 'M theory, orientifolds and G-flux', JHEP 9908 (1999) 023, hep-th/9908088.

[11] S. B. Giddings, S. Kachru, J. Polchinski, 'Hierarchies from fluxes in string compactifications', Phys. Rev. D66 (2002) 106006, hep-th/0105097.

[12] R. Blumenhagen, D. Lust, T. R. Taylor, 'Moduli stabilization in chiral type IIB orientifold models with fluxes', Nucl. Phys. B663 (2003) 319, hep-th/0303016.

[13] E. Witten, 'An SU(2) anomaly', Phys. Lett. B117 (1982) 324.

[14] C. Bachas, 'A Way to break supersymmetry', hep-th/9503030.

[15] R. Blumenhagen, L. Goerlich, B. Kors, D. Lust, 'Noncommutative compactifications of type I strings on tori with magnetic background flux', JHEP 0010 (2000) 006, hepth/0007024.

[16] C. Angelantonj, I. Antoniadis, E. Dudas, A. Sagnotti, 'Type I strings on magnetized orbifolds and brane transmutation', Phys. Lett. B489 (2000) 223, hep-th/0007090.

[17] G. Aldazabal, S. Franco, Luis E. Ibanez, R. Rabadan, A.M. Uranga, 'D $=4$ chiral string compactifications from intersecting branes', J. Math. Phys. 42 (2001) 3103, hepth/0011073.

[18] A. Sen, 'F theory and orientifolds', Nucl. Phys. B475 (1996) 562, hep-th/9605150,

[19] M. R. Gaberdiel, B. Zwiebach, 'Exceptional groups from open strings', Nucl. Phys. B518 (1998) 151, hep-th/9709013.

[20] A. Sen, 'Dynamics of multiple Kaluza-Klein monopoles in M and string theory', Adv. Theor. Math. Phys. 1 (1998) 115, hep-th/9707042.

[21] K. Becker, M. Becker, 'Supersymmetry breaking, M theory and fluxes', JHEP 0107 (2001) 038, hep-th/0107044.

[22] C. Vafa, 'Evidence for F theory', Nucl. Phys. B469 (1996) 403 hep-th/9602022.

[23] P. S. Aspinwall, 'K3 surfaces and string duality', hep-th/9611137. 
[24] R. Blumenhagen, V. Braun, B. Kors, D. Lust, 'Orientifolds of K3 and Calabi-Yau manifolds with intersecting D-branes', JHEP 0207 (2002) 026, hep-th/0206038.

[25] O. DeWolfe, B. Zwiebach, 'String junctions for arbitrary Lie algebra representations', Nucl. Phys. B541 (1999) 509, hep-th/9804210.

[26] D.-E. Diaconescu, G. W. Moore, E. Witten, 'A Derivation of K theory from M theory', hep-th/0005091; 'E(8) gauge theory, and a derivation of $\mathrm{K}$ theory from M theory', Adv. Theor. Math. Phys. 6 (2003) 1031, hep-th/0005090.

[27] E. Witten, 'Toroidal compactification without vector structure', JHEP 9802 (1998) 006, hep-th/9712028.

[28] A. R. Frey, J. Polchinski, 'N=3 warped compactifications', Phys. Rev. D65 (2002) 126009, hep-th/0201029.

[29] S. Kachru, M. B. Schulz, S. Trivedi, 'Moduli stabilization from fluxes in a simple IIB orientifold', JHEP 0310 (2003) 007, hep-th/0201028. 